\title{
A UTILIZAÇÃO DA SIMULAÇÃO BASEADA NA WEB PARA O ESTUDO DE PROCESSOS OPERACIONAIS
}

\section{THE USE OF WEB BASED SIMULATION FOR THE OPERATION PROCESS STUDY}

\section{Ilan Chamovitz}

Doutorando em Engenharia de Produção - COPPE/UFRJ

Universidade Federal do Rio de Janeiro

Cidade Universitária - C. T. - Blc. F - S. 105 - Rio de Janeiro - RJ - 21945-970

(21) 2562-8244 - e-mail: ilan@ufrj.br

\section{Francisco Santos Sabbadini}

Doutorando em Engenharia de Produção - COPPE/UFRJ

Universidade Federal do Rio de Janeiro

Cidade Universitária - C. T. - Blc. F - S. 105 - Rio de Janeiro - RJ - 21945-970

(21) 2562-8244 - e-mail: sabba@pep.ufrj.br

\section{Mário Jorge Ferreira de Oliveira \\ Professor Associado II, $\mathrm{PhD}$}

Universidade Federal do Rio de Janeiro

Cidade Universitária - C. T. - Blc. F - S. 105 - Rio de Janeiro - RJ - 21945-970

(21) 2562-8244 - e-mail: mario_jo@pep.ufrj.br 


\title{
RESUMO
}

A simulação baseada na Web provê recursos para a representação de alguns aspectos da realidade e inclui basicamente duas áreas: simulação e World Wide Web (WEB). A simulação pode ser aplicada para simplificar um modelo durante estudos de processos operacionais. A Web oferece um canal que pode ser usado para desenvolvimento, teste e disseminação de processos operacionais. O crescimento contínuo da Internet provoca mudanças: modelos podem ser construídos colaborativamente, via Web, e diversos recursos podem ser compartilhados por um numero significativo de usuários. Este trabalho apresenta diversos aspectos da simulação baseada na Web e sugere algumas tendências relacionadas ao estudo de processos operacionais.

Palavras-chave: Simulação baseada na Web, Simulação, Processos operacionais, Web, Pesquisa Operacional.

\begin{abstract}
The Web-Based Simulation provides resources for representing some reality aspects and includes basically two areas: Simulation and the World Wide Web(WEB). Simulation can be applied to simplify a model during operational process studies. Web offers a channel that can be used for processes operation development, testing and dissemination. The continued growing of the Internet provides changes: models turned to be built in a cooperative construction way, via Web, and several of them can be reached by a comprehensive number of users. This work presents several aspects of Web-based Simulation and suggests some course of actions related to Process Operation studies.
\end{abstract}

Key-words: Web based simulation, simulation, operation process, Web, Operational Research

\section{1 - INTRODUÇÃO}

A simulação baseada na Web abrange aspectos da Web e da simulação. A Web tem tido um impacto significativo no campo da computação em geral e no campo da simulação em particular. A simulação pode ser definida como a reprodução de um processo do mundo real ou de um sistema, durante um período de tempo. A crescente facilidade de conexão, a diminuição do custo de acesso e a disseminação do conhecimento utilizando-se as novas tecnologias de informação e comunicação promovem, com o passar dos anos, mudanças nos comportamentos de pesquisadores, professores, alunos e dos profissionais em empresas. Fica cada vez mais fácil um gerente controlar e gerir a produtividade de sua equipe enquanto realiza uma viagem internacional. Grupos de executivos trocam experiências, utilizando um 
fórum virtual, sobre melhores práticas em um determinado segmento de negócio. Alunos preparam trabalhos de forma cooperativa utilizando um gerenciador de mensagens. A tecnologia já permite a realização de atividades em laboratórios virtuais, independente da localização de pessoas e de instrumentos. Por exemplo, após o preenchimento de um formulário na web, pode-se agendar uma observação astronômica remota, via Internet (INPE 2006).

Neste contexto de mudanças, em 1996, a partir de 3 artigos (FISHWICK, 1996; BUSS \& STORK, 1996; NAIR et. al., 1996) apresentados em uma sessão técnica do Winter Simulation Conference, surge um novo campo de estudos, a simulação baseada na Web que compreende aspectos técnicos e operacionais de natureza diversa, que serão especificados ao longo do artigo, e tornam este tema interessante, abrangente e difuso.

Esta pesquisa não pretende esgotar o tema, mas contribuir para o entendimento acerca da simulação baseada na Web e apontar algumas tendências para o futuro de sua aplicação em estudos de processos operacionais. A pesquisa é exploratória, essencialmente qualitativa, e foi realizada em 2006, durante a disciplina de Tópicos Especiais em Simulação, em um curso de pós-graduação stritu sensu de uma universidade federal. A metodologia utilizada é descrita na seção 2. A seção 3 apresenta o conceito e a relevância da simulação baseada na Web. A seção 4 descreve o surgimento da web simulation na Pesquisa Operacional. A seção 5 é dedicada à simulação, ambientes de desenvolvimento e são apresentadas algumas aplicações disponíveis na Web. Na seção 6 são apresentados os temas e as áreas de aplicação. Na seção 7 são apresentadas as tendências para o futuro da Web-based simulation e as conclusões finalizam o artigo, na seção 8 .

\section{2 - METODOLOGIA}

A pesquisa foi estruturada seguindo uma metodologia específica de busca utilizada anualmente e apresentada por um dos autores durante o curso da disciplina citada, no Programa de Engenharia de Produção, com o objetivo de acompanhar a evolução de determinadas áreas da Pesquisa Operacional, no campo da simulação a eventos discretos. A proposta metodológica é de que alunos do ano corrente pesquisem e complementem as informações já organizadas pelos colegas do período anterior, de modo a formar um repositório de conhecimento em permanente atualização. 
Para este artigo, o processo de investigação foi realizado para coletar e estruturar informações sobre Web-Based Simulation, identificada como um campo novo de estudo e ainda em desenvolvimento, englobando recursos tecnológicos da Web combinados com a tecnologia de simulação. A metodologia utilizada compreende 5 aspectos: 1) sobre as organizações de pesquisa, envolvidas com a área pesquisada; 2) os principais pesquisadores que desenvolvem projetos; 3) as conferências realizadas ou agendadas. 4) dentro de cada conferência, identificam-se os temas englobados naquele ano e 5) para cada tema, quantos trabalhos foram selecionados. Uma vez quantificados por tema, os alunos preparam uma relação de títulos dos trabalhos publicados que consideram inovadores, com os nomes dos autores de cada trabalho. O material é analisado em aula pelos discentes e os documentos produzidos são armazenados no servidor do grupo de Pesquisa Operacional do Programa de Pós-graduação. Parte do material está disponível para acesso em http://api.adm.br/websimulation.htm. Para complementar o processo, o grupo realizou pesquisas na literatura e na Internet. As tendências são baseadas na verificação de novos temas e na evolução tecnológica. Para registrar e organizar os eventos pesquisados foi utilizada a técnica de Redes Sistêmicas (BLISS, MONK \& ORGBORN, 1983) e um software que permitiu registrar na Web, em um só local e de forma sistêmica, as informações sobre os sites pesquisados.

\section{3 -A IMPORTÂNCIA E A ABRANGÊNCIA DA SIMULAÇÃO BASEADA NA WEB}

Page \& Opper (1999) citam o princípio da separação de conceitos (DIJKSTRA, 1976), que afirma que o principal objetivo da maioria dos sistemas de software é a corretude. A corretude é baseada em se considerar primeiramente os aspectos de design do software, independentemente da infra-estrutura que servirá para a execução do sistema. A separação das etapas de modelagem e de execução promove mudanças nestas duas fases. Assim, o ambiente de simulação que oferece a infra-estrutura para a execução da simulação deve aceitar a modelagem previamente desenvolvida e permitir a sua implementação. São seis princípios que servem para diferenciar a simulação baseada na Web da tradicional, e servem para prever algumas mudanças no processo de simulação. Na tabela 1, adaptada de (PAGE \& OPPER, 1999), constam os princípios e algumas questões pertinentes. 
Tabela 1. Princípios e questões de simulação baseada na Web

\begin{tabular}{|c|c|}
\hline $\begin{array}{c}\text { Princípios para simulação na } \\
\text { web }\end{array}$ & Questões pertinentes \\
\hline Proliferação de objetos digitais & $\begin{array}{l}\text { Identificação de responsáveis pelos objetos, problemas de } \\
\text { segurança, direitos de propriedade e de uso dos objetos; } \\
\text { excesso de objetos disponíveis, problemas de validação e } \\
\text { pesquisa. }\end{array}$ \\
\hline $\begin{array}{l}\text { Disseminação de padrões de } \\
\text { software }\end{array}$ & $\begin{array}{l}\text { Importante para a interoperabilidade de aplicações e para a } \\
\text { modelagem ou uso de componentes. Ex. CORBA, UML, } \\
\text { OLE/COM, HLA. }\end{array}$ \\
\hline Construção por composição & $\begin{array}{l}\text { Aplicações podem ser composições de objetos digitais, } \\
\text { acessíveis via web. Apenas funcionalidades não existentes } \\
\text { devem ser codificadas. }\end{array}$ \\
\hline $\begin{array}{l}\text { Aumento da abordagem } \\
\text { "tentativa e êrro", }\end{array}$ & $\begin{array}{l}\text { Desenvolvimento da modelagem ganha velocidade: } \\
\text { modelo de simulação pode ser testado, alterado e } \\
\text { novamente testado... }\end{array}$ \\
\hline \begin{tabular}{|l} 
Proliferação de uso de \\
simulação por leigos
\end{tabular} & $\begin{array}{l}\text { Com a disseminação de componentes via web, pessoas } \\
\text { despreparadas têm acesso e podem utilizá-los de forma } \\
\text { indevida. }\end{array}$ \\
\hline $\begin{array}{l}\text { Arquitetura multi-camada e } \\
\text { sistemas multi-linguagens }\end{array}$ & $\begin{array}{l}\text { Em uma simulação, componentes podem ser } \\
\text { desenvolvidos para construir modelos para execução } \\
\text { repetitiva, e outra linguagem pode ser utilizada para } \\
\text { auxiliar na execução dos modelos em tempo real. }\end{array}$ \\
\hline
\end{tabular}

Fonte: Adaptação a partir de Page e Opper (1999)

A Simulação baseada na Web foi classificada por Page e Opper (1999) em cinco áreas:

- Simulação como hipermídia: A produção, armazenamento e recuperação de documentos textuais, imagens, áudio e vídeo utilizando a Internet oferece facilidades para o treinamento a distância, educação e capacitação baseada em simulação.

- Metodologia da Pesquisa em Simulação: modelos podem ser rapidamente disseminados na Web. A publicação de artigos e a divulgação de resultados modificam o comportamento de pesquisadores, professores e estudantes. Novas abordagens para realização de pesquisa são estudadas e implementadas.

- Acesso baseado em Web para programas de simulação: esta área inclui a execução remota de simuladores hospedados em um computador servidor de aplicações, utilizando navegadores (browsers) e, também, códigos que podem executar simulações em computadores-cliente.

- Modelagem e simulação distribuída: esta área inclui atividades que usam tecnologias orientadas para Web, tais como CORBA, Java e RMI, como infra-estrutura para suportar a execução de simulação distribuída. Jogos com Internet e plataformas para 
cooperação no desenvolvimento e modelagem de simuladores também estão incluídos nesta área.

- Simulação na Web: Modelagem e análise da Web para otimização e performance estão incluídas nesta área.

Os autores não limitam apenas a estas categorias, mas percebemos que muitos trabalhos podem ser classificados em uma ou mais, inclusive com superposição das mesmas. Isto pode ser apreciado ao identificarmos os temas que fizeram parte da primeira conferência Internacional de Modelagem e Simulação baseadas na web (WEBSIM98, 1998).

\section{4 - HISTÓRICO DA WEB E DA SIMULAÇÃO BASEADA NA WEB}

A Web foi criada a partir de um projeto da CERN (Organização Européia para Pesquisa Nuclear), chamado ENQUIRE, iniciado por Tim Berners-Lee em 1989. Baseado no conceito de hipertexto, o projeto visava, principalmente, permitir que pesquisadores compartilhassem informações. O primeiro web site foi ao ar em 1991. Em 30 de abril de 1993, foi anunciado pela CERN que a Web seria gratuita para todos. A década de 90 foi marcada pela evolução da WWW. Em 1995 houve um esforço grande, a partir do desenvolvimento de diversos grupos de trabalho e, como consequiência, o ano de 1996 foi marcado por inovações, pela versão HTML 3.2 da linguagem de hipertexto. Nesta versão foram adicionados diversos recursos como tabelas, applets e texto em torno de imagens, sobrescrito e subscrito.

Neste mesmo ano (1996), foram apresentados os primeiros artigos sobre web-based simulation numa sessão técnica na Winter Simulation Conference, inicialmente como parte da área temática de metodologia de modelagem. A partir de 2000 passa a ser uma área especifica da WSC. O interesse crescente e a relevância do tema ficam evidentes com a criação em 1998 da primeira Conferência Internacional de Modelagem e Simulação baseada em Web. Além disso, com base nos arquivos da International Modeling and Simulation Multiconference, identificou-se a Web-Simulation como uma seção temática específica, da Annual Simulation Symposium (ANSS) onde se podem encontrar diversas abordagens como em Huang e Miller (2001), Holmes et al (2001), Belfore et al (2001), Holmes et al (2002), Miller et al (2002), Lima Filho e Hirata (2002), Bruzzone e Orsoni (2003), Truong (2004), Huang e Madey 
(2005). De 1999 até 2005, os principais pesquisadores envolvidos com o tema desenvolveram simuladores e muitos estão disponíveis na Internet até hoje. O anexo I, apresenta uma compilação das principais áreas, pesquisadores e instituições envolvidas com a simulação baseada na Web. A tabela 2 apresenta uma síntese cronológica baseada nos trabalhos apresentados nas edições da WSC de 1996 a 2005 e da ANSS de 2001 a 2005.

Tabela 2. Síntese do desenvolvimento da Web-Based Simulation

\begin{tabular}{|c|c|c|}
\hline Ano & Posicionamento & Tópico de sessão temática \\
\hline 1996 & Tópico de sessão temática & $\begin{array}{l}\text { Conceitos, Simulação a eventos discretos na Web e Linguagem Java para } \\
\text { ambiente de simulação }\end{array}$ \\
\hline 1997 & Tópico de sessão temática & $\begin{array}{l}\text { Web-Based Simulation baseada em Java, Linguagem de simulação de } \\
\text { processos baseada em Java e Modelagem de filas e redes baseada em Java. }\end{array}$ \\
\hline 1998 & Tópico de sessão temática & Arquitetura, Método das três fases em Java e Ambientes de simulação \\
\hline 1999 & Tópico de sessão temática & $\begin{array}{l}\text { Simulação em rede baseada na Web (CORBA), Arquitetura, processamento } \\
\text { de dados on-line em modelos de simulação e visualização de simulação a } \\
\text { eventos discretos distribuída }\end{array}$ \\
\hline 2000 & $\begin{array}{l}\text { Sessão temática } \\
\text { abordandoWeb-Based e } \\
\text { Java-Based Simulation }\end{array}$ & $\begin{array}{l}\text { Otimização da simulação a eventos discretos distribuída baseada na Web, } \\
\text { simulação de sistemas distribuídos baseada na Web, VRML e motor de } \\
\text { busca baseado em Java para UML. }\end{array}$ \\
\hline 2001 & $\begin{array}{l}\text { Sessão temática especifica } \\
\text { Web-Based Simulation }\end{array}$ & $\begin{array}{l}\text { Aplicações em Supply Chain e APL, simulação paralela, sistema de } \\
\text { treinamento }\end{array}$ \\
\hline 2002 & $\begin{array}{l}\text { Sessão temática especifica } \\
\text { Web-Based Simulation }\end{array}$ & $\begin{array}{l}\text { Suporte para a simulação de projetos, Aplicações em manufatura, } \\
\text { Linguagem SRML ( Simulation Reference Markup Language) e simulador } \\
\text { SR ( Simulation Reference Simulator), Web Services, Projetos de simulação } \\
\text { e B2B. }\end{array}$ \\
\hline 2004 & $\begin{array}{l}\text { Sessão temática } \\
\text { renomeada } \\
\text { Tecnologias de simulação } \\
\text { possibilitadas pela WEB } \\
\text { dividida em 03sub áreas } \\
\text { temáticas: 1) Governo e } \\
\text { Defesa; 2) Ferramentas e } \\
\text { aplicações em XML; } \\
\text { 3)Ferramentas, linguagens } \\
\text { eaplicações possibilitadas } \\
\text { pela WEB. }\end{array}$ & $\begin{array}{l}\text { Arquitetura de sistema de alto nível, simulação paralela a eventos discretos, } \\
\text { ambientes de simulação baseada em XML, modelagem de simulação, } \\
\text { simulação Supply Chain baseada em XML. }\end{array}$ \\
\hline 2005 & 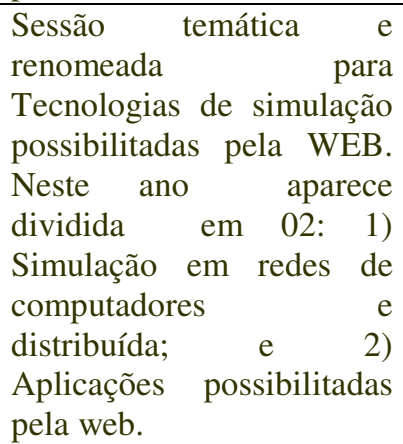 & $\begin{array}{l}\text { Simulação de infra-estrutura de redes de computadores, consistência de } \\
\text { dados em infra-estrutura de alto desempenho, modelos de simulação on- } \\
\text { line, modelagem e simulação de sistemas complexos baseados em XML. }\end{array}$ \\
\hline
\end{tabular}

Fonte: WSC (1995-2005) e ANSS (2001-2005)

Para facilitar o acesso aos principais trabalhos desenvolvidos envolvendo simulação baseada na Web, os autores estão construindo redes sistêmicas (BLISS, MONK \& 


\section{Revista Produç@o}

Associação Brasileira de Engenharia de Produção - ABEPRO

Universidade Federal de Santa Catarina - UFSC

www.producaoonline.org.br

ISSN 1676 - 1901 / Vol. VIII/ Num. IV/ 2008

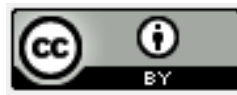

ORGBORN, 1983) para estruturar e organizar conceitos sobre o assunto. A ferramenta utilizada para construção das redes é o GRS - Gerador de Redes Sistêmicas na Web (CHAMOVITZ \& ELIA, 2003). Para acessar as redes basta acessar o programa no endereço do Projeto GRS e digitar o código das redes (396 e 397) no campo de atalho. A rede 396 contém links para os sites dos principais eventos de Simulação na Web, estruturada por ano de realização do evento. A rede 397 apresenta as principais categorias relacionadas com Simulação baseada na Web. Para conhecer melhor as Redes Sistêmicas e o GRS acesse http://www.nce.ufrj.br/ginape/GRS . Para acessar o programa clique no link GRS, localizado no menu lateral da página principal do projeto. A figura 1 apresenta a tela do GRS e a rede 396, com algumas páginas abertas a partir do clique nos links dos eventos realizados desde o ano de 1998.

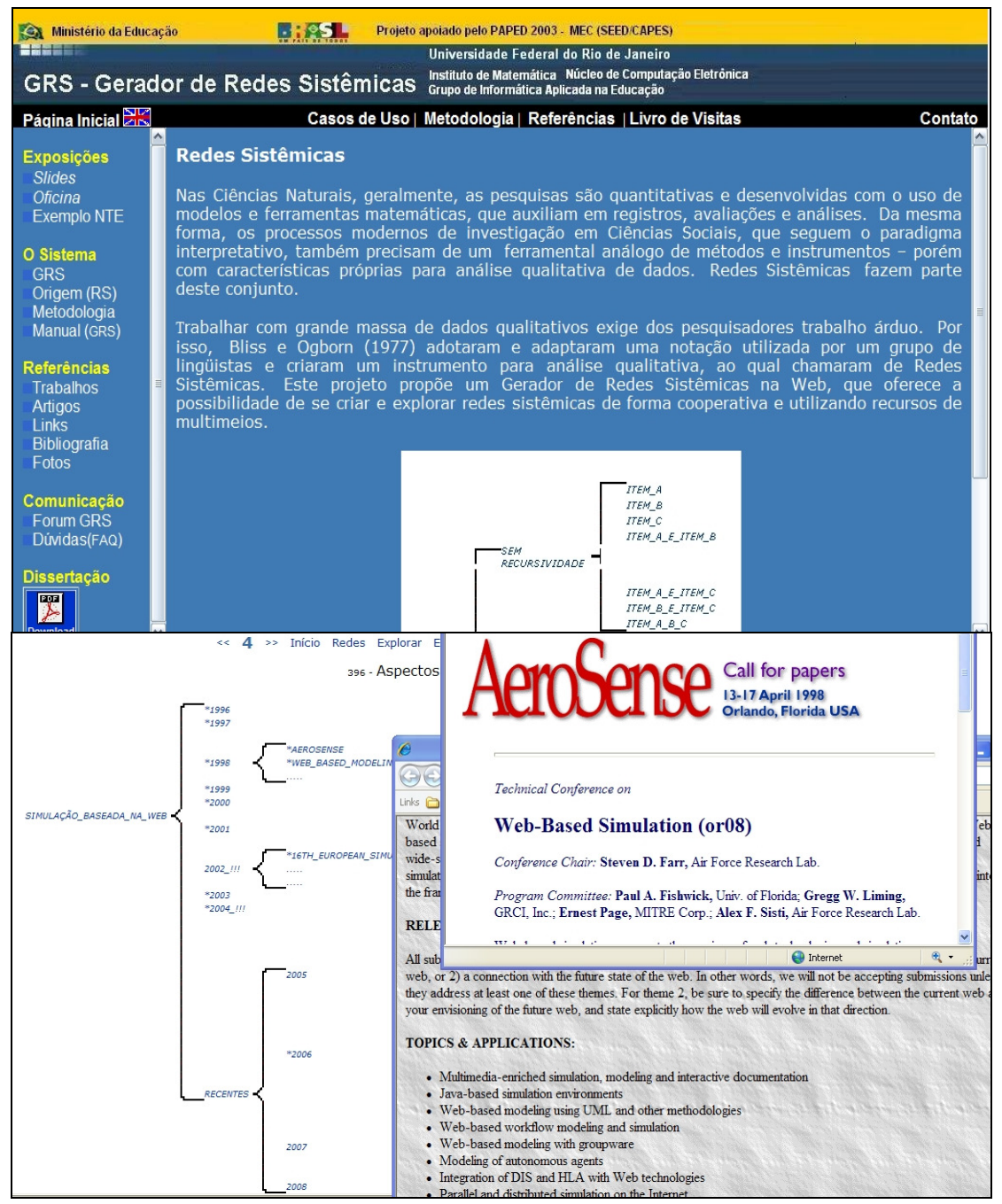

Figura 1. Tela do projeto GRS e a rede sistêmica 396

Fonte: http://www.api.adm.br/GRS 


\section{5 - SIMULAÇÃO E AMBIENTES DE DESENVOLVIMENTO}

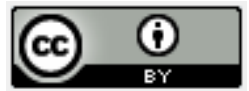

Alguns modelos de simulação podem ser desenvolvidos para serem executados no servidor - um computador central, que coloca disponível o aplicativo. Outros modelos são executados na própria máquina do cliente, após serem copiados, via web. Maiores detalhes sobre a execução de simulação baseada na web podem ser recuperados no trabalho de Reichenthal (2002). O autor reintroduz a simulação baseada na web pelo ponto de vista do desenvolvedor de software e, também, apresenta uma linguagem e um simulador, ambos construídos em pesquisas na Boeing: a linguagem SRML - Simulation Reference Markup Language e o simulador SR (Simulation Reference simulator).

A utilização da abordagem de orientação a objetos revelou-se adequada para a construção de modelos de simulação baseada na Web. A construção de componentes de software, reutilizáveis, independentes de plataforma e escaláveis, permite o aumento da velocidade de desenvolvimento de software e uma minimização dos custos. A web oferece a oportunidade de colaboração e cooperação. Desenvolvedores disseminam conhecimentos e constroem, à distância e em grupo, componentes de software, que ficam disponíveis na web. Neste contexto, tecnologias baseadas em componentes facilitam a interoperabilidade.

A linguagem Java permite uma abordagem orientada a objeto, compatível com o ambiente de simulação. É independente de plataforma, é composta por módulos voltados para modelagem e simulação. Ambientes de desenvolvimento facilitam o desenvolvimento de componentes ou de aplicações: JBuilder, da Borland, é um exemplo. A Microsoft e a Sun também construíram ambientes para desenvolvimento em Java, que permitem, em diversos casos, a produção de software utilizando-se janelas, arrastando e soltando elementos gráficos, inserindo valores em caixas de texto, clicando em botões, sem a necessidade de se digitar código em editores de texto. Alguns ambientes permitem a geração do modelo graficamente e, com apenas alguns cliques, constroem a aplicação, gerando o código, e o programa, pronto para ser executado.

\section{6 - TEMAS ABORDADOS EM EVENTOS, ÁREAS DE APLICAÇÃO DA SIMULAÇÃO BASEADA NA WEB E APLICAÇÕES VOLTADAS PARA O ESTUDO DE PROCESSOS OPERACIONAIS}

A utilização da simulação em diversas áreas foi estendida, graças à utilização de plataformas baseadas na Web e de componentes, que permitem a distribuição e o reuso de 
código em programas de computador. A partir dos Programas dos Congressos em Simulação Baseada na Web foram coletados tópicos pertinentes, apresentados na tabela 3:

Tabela 3. Tópicos em Simulação Baseada na Web

- Metodologia e Ferramentas

- Computação de alta performance e grande escala

- Verificação, validação certificação

- Educação e jogos

- Simulação em meio ambiente, ecologia, biologia e medicina

- Ambiente de simulação baseada na Web (WSE)

- Simulação Interativa Distribuida baseada na web (WDIS)
- Técnicas e Padrões para integração de modelos

- Comunicação e Interoperabilidade em WSE and WDIS

- WSE e WDIS em aplicações de educação, treinamento e aprendizagem.

- Simulation visualization/animation in WSE and WDIS

- Simulação Distribuida baseada na Web

- Linguagens e ferramentas para simulação discreta

- Compartilhamento e reuso de modelos de simulação e ferramentas em ambientes de simulação baseada na web - WSE

Fonte: Winter Simulation Conference - WSC (1996-2005), Annual Simulation Symposium ANSS (2001-2005)

Diversas aplicações vêm sendo desenvolvidas e distribuídas via Web. A lista não pretende esgotar a totalidade das aplicações existentes e possíveis, mas tão somente contribuir para algumas compilações relacionadas ao tema. O sucesso de uma organização depende fundamentalmente da eficiência e da efetividade de seus processos de negócio. Neste sentido a combinação das tecnologias web e da simulação a eventos discretos contribui significativamente para o entendimento e a identificação de oportunidades de melhorias significativas nas operações e nos seus processos, compreendendo aspectos operacionais, táticos e estratégicos. Na tabela 4 estão relacionadas as principais áreas onde a simulação baseada na Web vem sendo utilizada. 
Tabela 4. Áreas de aplicação da Simulação Baseada na Web

\begin{tabular}{|c|c|}
\hline $\begin{array}{l}\text { - } \text { - Imagtemas automotivos } \\
\text { - } \text { Robótica } \\
\text { - } \text { Eletrônica, Informática e } \\
\text { Telecomunicações } \\
\text { - Logística } \\
\text { - Transportes } \\
\text { - } \text { - Restão da Saúde } \\
\text { gráficas em aplicações industriais e } \\
\text { - } \text { Aquerciais } \\
\text { - } \text { representação do conhecimento } \\
\text { - Podelagem cognitiva } \\
\text { - Problema de tomada de decisões } \\
\text { - Visãa de teoremas }\end{array}$ & $\begin{array}{l}\text { - } \text { Modelagem de Sistemas Complexos } \\
\text { - } \text { Aeroespacial } \\
\text { - } \text { - } \text { Desininha } \\
\text { - } \text { Engenharia de Processos } \\
\text { - Energia } \\
\text { - Engenharia Petroquímica } \\
\text { - Militar } \\
\text { - Verificação, validação e certificação } \\
\text { - Educação, treinamento } \\
\text { - } \text { Modelagem do desempenho humano } \\
\text { - } \text { Planejamento } \\
\text { - Raciocínio aproximado } \\
\text { - Reconhecimento de padrões }\end{array}$ \\
\hline
\end{tabular}

Fonte: Winter Simulation Conference - WSC (1996-2005), Annual Simulation Symposium ANSS (2001-2005)

Métodos estatísticos são utilizados para programação da produção, acompanhamento de vendas, auditoria, controle de processos operacionais, na indústria, comércio e em serviços. Para estudar os modelos estatísticos, por exemplo, o Rice Virtual Lab in Statistics pode ser acessado no endereço http://www.ruf.rice.edu/ lane/rvls.html, e oferece aplicativos com demonstrações estatísticas: ANOVA, distribuição binomial, Chi-Quadrado, Intervalo de Confiança, Distribuições, Teste T, entre outras.

Alguns autores também oferecem páginas na web com uma lista de aplicativos e modelos desenvolvidos por seus grupos ou explorados em outros sítios. $\mathrm{Na}$ página do professor Fishwick (http://www.cise.ufl.edu/ fishwick/websim.html) existem vários exemplos de demonstrações e simulações: Simulação de Monte Carlo híbrida, tráfego rodoviário, transporte ferroviário, entre outras. Na figura 2 é apresentada a aplicação que simula o tráfego metroviário na cidade de Los Angeles, Califórnia - Estados Unidos. 


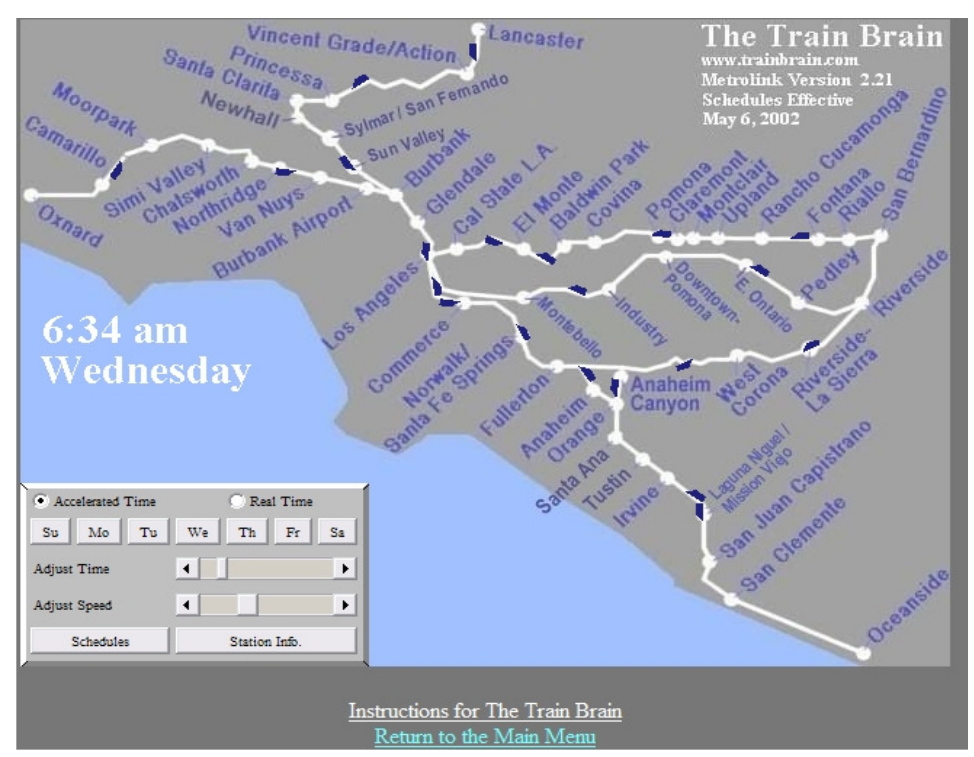

Figura 2. Metrolink

Fonte: http://www.trainbrain.com/metrolink.html

Outra fonte de aplicações e assuntos relacionados a simulação baseada na web é a página do projeto MITRE, finalizado em 1999. A página, apesar de não estar sendo atualizada, apresenta várias referências e pode ser acessada a partir do endereço http://www.thesimguy.com/Projects/websim/survey/text.html. Alguns modelos podem ser executados em servidores centrais por meio de programas instalados nos servidores Common Gateway Interface (CGI), outros são executados no cliente, ou seja, na própria máquina do usuário, utilizando applets Java.

Aplicações em Web-Based Simulation voltadas para processos operacionais podem ser encontradas em diversas áreas, algumas das quais serão apresentadas a seguir. Lulay e Reinhart (1998), apresentam a utilização das tecnologias Web associadas a um modelo hierárquico de simulação, para a tomada de decisão com o objetivo de agilizar o processamento de pedidos e aumentar a flexibilidade em unidades de produção descentralizadas. A partir desse trabalho os autores identificaram a customização do modelo pelo uso dos recursos tecnológicos combinados (Web e simulação) e desenvolveram um sistema assistente de simulação para monitoramento da produção, contribuindo para a tomada de decisão locais em consonância com os objetivos globais, relacionados à unidades de produção descentralizadas.

Seila e Miller (1999) apresentam uma aplicação baseada em Web-Based Simulation para avaliação de desempenho e tomada de decisão. Neste estudo os autores avaliaram um 


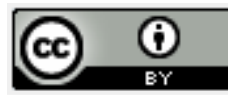

serviço de emergência hospitalar sob diversos aspectos e detalham os passos e requisitos relativos ao modelo de simulação e ao ambiente de desenvolvimento do mesmo.

Chandrasekaran et al (2002) apresentam um estudo relacionando serviços Web com simulação, no qual utilizam estas tecnologias para a avaliação de um serviço de compras pela internet através da simulação dos processos com o objetivo de identificar oportunidades de melhoria. Os autores desenvolveram uma ferramenta, Web Services Designer Tool (WSDT), a qual associada ao software de simulação JSIM, permite acompanhar visualmente os processos Web em ação antes da sua implementação, possibilitando um melhor entendimento das operações.

Em projetos de simulação há necessidade de atividades cooperativas que envolvem uma intensa comunicação entre os participantes. Henriksen et al (2002) apresentam o Web Based Simulation Center (WBSC) que foi desenvolvido com o intuito de dar suporte, a partir de ferramentas disponíveis na Web, a especialistas de vários campos envolvidos em projetos de simulação. O conceito compreende o desenvolvimento e o gerenciamento de projetos de simulação de forma colaborativa na qual consultores e desenvolvedores distintos, separados geograficamente, podem atuar de maneira interativa e operacionalmente. Em colaboração com universidades, o protótipo do software WBSC foi aplicado em um projeto de simulação B2B.

Graupner et al (2002) utilizaram Web-Based simulation e animação para auxiliar o trabalho de equipes de vendas no acompanhamento dinâmico da demanda individualizada de produtos em função das necessidades dos consumidores. A ferramenta desenvolvida permite configurar, simular e animar um sistema de manufatura via internet, funcionando como um sistema de apoio à decisão para estes profissionais. Após a simulação é possível visualizar o modelo em 2D/3D além de avaliar a performance do sistema.

Moraes et al. (2002) utilizaram os recursos tecnológicos da Web para uma simulação virtual multiusuário, na qual estudam o processo de chegada de pacientes num hospital e o comportamento das filas.

Uma aplicação utilizando técnicas de simulação paralela a eventos discretos é descrita por Rabelo et al. (2004), com o objetivo de representar o paralelismo funcional estruturado presente no fluxo de operações relacionadas ao lançamento de naves do sistema de transportes da NASA. Os autores apresentam um ambiente paralelo síncrono para emolução e simulação a eventos discretos que permite simular o ciclo de vida operacional do lançamento espacial e possibilita a customização utilizando Java 3D e VRML (Virtual Reality Modeling Language). 
Uma aplicação envolvendo simulação da cadeia de suprimentos pode ser vista em Chatfiel et al.(2004) e em operações espaciais da NASA em Park (2005).

\section{7 - TENDÊNCIAS PARA O FUTURO}

A Web 2.0 reforça alguns conceitos, baseados em colaboração. Usuários passam a ser mais responsáveis pelo conteúdo da web. Aplicativos que hoje estão nos computadores de cada indivíduo, passam a ficar disponíveis na Web. Assim, com apenas o sistema operacional e o navegador, um aluno pode utilizar um editor de texto que está disponível na Web e salvar o texto produzido em um servidor web, acessível por um endereço web (URL). Estando em outro ambiente físico ou em outro país, um colega pode alterar o arquivo e salvá-lo também. Este procedimento vale para planilhas, bancos de dados e aplicativos. Vale também para desenvolvimento de modelos de simulação. E mais: após construir o modelo, o responsável pode colocá-lo na web, disponível para teste.

A partir da pesquisa efetuada foram identificadas algumas tendências para o futuro da Web-Base simulation, compreendendo particularmente esforços de institutos de pesquisa, empresas e universidades no sentido melhorias nas seguintes áreas:

- Arquitetura de alto desempenho, construção de ambientes de simulação baseados em recursos tecnológicos da Web, Simulação de operações on-line, em tempo real e com a captação de dados para a execução de simulações a partir da internet;

- Aplicações em 3D, visualização em 3D, modelagem e animação via internet e realidade virtual;

- Desenvolvimento de ferramentas, plataformas, linguagens e simuladores baseados nas tecnologias da Web;

- Modelagem flexível, ambiente gráfico integrado;

- Multi-modelagem e simulação em ambiente colaborativo e em tempo real.

- Simulação distribuída e paralela a eventos discretos;

- Sistemas de treinamento de simulação,via Web; e

- Simulação virtual multiusuário baseada na Web. 


\section{8 - CONCLUSÕES}

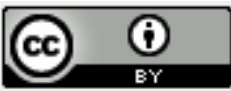

A pesquisa realizada demonstra a amplitude, a relevância e as oportunidades relacionadas com a simulação baseada na Web, considerando-se o fórum de debates, os pesquisadores, as instituições envolvidas com o tema, as áreas de aplicação identificadas e particularmente no que diz respeito ao estudo e ao entendimento de processos operacionais. Neste campo a WebBased simulation contribui significativamente como ferramenta de apoio à decisão com implicações gerenciais e estratégicas importantes. O crescimento do acesso via web, a disseminação de componentes e aplicativos com modelos e simuladores cresce cada vez mais. Dentre os benefícios oferecidos se encontra a possibilidade da construção de modelos em cooperação, o compartilhamento de softwares e a construção coletiva do conhecimento que resultam numa maior compreensão dos processos, mais agilidade, eficiência e eficácia para os gestores. No mesmo sentido contribuem efetivamente para a solução de problemas. Quanto aos riscos podemos considerar que muitos problemas com a utilização de simuladores na Web estão relacionados com o excesso de informação, a necessidade de validação de grande quantidade de componentes disponíveis e da informação oferecida.

\section{REFERÊNCIAS}

BERNERS-LEE,T (1990) - Information Management: A Proposal. CERN, March 1989, May 1990.Diponivel em http://www.w3.org/History/1989/proposal.html. Acesso em junho de 2006.

BELFORE, L.A; CHITITHOTI, S. Multiuser extensions to the interactive Land Use VRML application (ILUVA). Proceedings of the 34th Annual Simulatin Symposium, 2001.

BLISS, J., MONK, M., OGBORN, J. Qualitative Data Analysis for Educational Research: A guide of systemic networks. London, 1983. Croom Helm.

BRUZZONE, A.; ORSONI, A. AI and simulation-based techniques for the assessment of supply chain logistic performance. Proceedings of the $36^{\text {th }}$ Annual Simulatin Symposium, 2003.

BUSS, A.R.; STORK, K.A. Discrete-event simulation on the World Wide Web using Java. Proceedings of the 1996 Winter Simulation Conference .

CHAMOVITZ, I., ELIA, M.F.. Gerador de Redes Sistêmicas: um instrumento de apoio a pesquisa na Web. Anais do Simpósio Brasileiro de Informática na Educação. Rio de Janeiro: Sociedade Brasileira de Computação, 2003. v.1. p.803 - 804. [Mostra de Software]. 


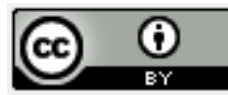

CHANDRASEKARAN S., SILVER, G., MILlER J. A., CARDOSO J. S., SHETH A. P. Web Service Technologies and their Synergy with Simulation. Proceedings of the 2002 Winter Simulation Conference.

CHATFIEL, D.C.; HARRISON, T.P. e HAYYA, J.C. XML-Based supply chain simulation modeling. Proceedings of the 2004 Winter Simulation Conference .

DIJKSTRA, E.W.(1976). A discipline of Programming, Prentice-Hall, Englewood Cliffs, NJ.

FISHWICK, P. A. Web-Based simulation: some personal Observations. Proceedings of the 1996 Winter Simulation Conference.

GRAUPNER, T.; RICHTER, H. e SIHN, W. Configuration, simulation and animation of manufacturing system via internet. Proceedings of the 2002 Winter Simulation Conference.

HENRIKSEN, J.O.; LORENZ, P; HANISCH, A. OSTERBURG, S. SCRIBER, T.J. Web Based Simultion Center: professional support for simulation projects. Proceedings of the 2002 Winter Simulation Conference.

HOLMES, V.P.; LINEBARGER, J.M.; MILER, D.J. VANDERWART, R.L. Envolving the Web-based distributed SI/PDO Architecture for High-Performance Visualisation. Proceedings of the $34^{\text {th }}$ Annual Simulatin Symposium, 2001.

HOLMES, V.P.; KLEBAN, S.D.; MILLER, D.J.; PAVLAKOS, C.; POORE, C.A., VANDERWART, R.L. An architecture and implementation to support large-scale data access in scientific simulation environments. Proceedings of the $35^{\text {th }}$ Annual Simulatin Symposium, 2002.

HUANG, X ; MILLER, J.A. Building a Web-Based Federated Simulation System with Jini and XML. Proceedings of the $34^{\text {th }}$ Annual Simulatin Symposium, 2001.

HUANG, Y.; MADAY, G. Autonomic Web-Based simulation. Proceedings of the $38^{\text {th }}$ Annual Simulatin Symposium, 2005.

INPE (2006) Intituto de Pesquisas Espaciais - Miniobservatório Astronômico. Disponível em http://www.das.inpe.br/miniobservatorio/index.php. Acesso em junho de 2006.

LULAY, W.E.; REINHART, G. Coordinating order processing in descentrilized production units using hierarquical simulaton models and web-technologies. Proceedings of the 1998 Winter Simulation Conference.

LIMA FILHO, H.A.S.; HIRATA, C.M. GroupGraph: a collaborative hierarchical graph editor based on the internet. Proceedings of the $35^{\text {th }}$ Annual Simulatin Symposium, 2002.

MILLER, J.A.; CARDOSO, J; SUKVER, G. Using simulation to facilitate effective workflow adaptation. Proceedings of the $35^{\text {th }}$ Annual Simulatin Symposium, 2002. 


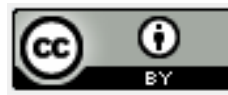

MILLS, JD (2002) Using Computer Simulation Methods to Teach Statistics: A Review of the Literature. Journal of Statistics Education Volume 10, Number 1 (2002). Disponível em http://www.amstat.org/publications/jse/v10n1/mills.html. Acesso em junho de 2006.

MORAES, A.B.; DE OLIVEIRA, M.J.F.; ESPOSITO, S.M.; BORDALO, S.M. A multi-user simulation of a hospital queue. Proceeding of the 28th Meeting of the European Working Group onOperational Research Applied to Health Services, 2002.

NAIR, R. S.; MILLER, J. A.; ZHANG, Z. A Java-Based Query Driven Simulation Environment. Proceedings of the 1996 Winter Simulation Conference.

PAGE E.H. (1998) The Rise of Web-Based Simulation: Implications for the High Level. Proceedings of the 1998 Winter Simulation Conference.

PAGE, E.H., Opper, J.M, (1999). Investigating the Application of Web-based Simulation Principles within the Architecture for a Next-Generation Computer Generated Forces Model. To appear in Future Generation Computer Systems, Elsevier Science Publishing

PAGE, E.H., BUSS, A., FISHWICK, P.A., HEALY, K.J., NANCE, R.E. AND PAUL, R.J. (1999). "Web-Based Simulation: Revolution or Evolution?" submitted to: ACM Transactions on Modeling and Computer Simulation, February.

PARK, J. MORAGA, R; RABELO, L.; DAWSON, J.; MARIN, M e SEPULVEDA, J. Addressing complexity using distributed simulation: a case study in spaceport modeling. Proceedings of the 2005 Winter Simulation Conference.

RABELO, L.; SEPULVEDA, J.; MARIN, M.; PARUCHURI, A.; NAYARANAN, K. Parallel discrete event simulation of space shuttle operations. Proceedings of the 2004 Winter Simulation Conference.

REICHENTHAL, S.W. Re-introducing Web-based simulation. Proceedings of the 2002 Winter Simulation Conference.

SEILA, A.F.; MILLER, J.A. Scenario management in Web-Based Simulation. Proceedings of the 1999 Winter Simulation Conference.

TRUONG, T.N. Computational science and engineering on-line: an integrated web-based simulation environment for computational science and engineering. Proceedings of the $37^{\text {th }}$ Annual Simulation Symposium, 2004.

WDVL (2006) - Web Developers Library. History of the Internet and the World Wide Web. Disponível em http://wdvl.internet.com/Internet/History/ . Acesso em junho de 2006.

WEBSIM98 (1998) - International Conference on Web-based Modeling \& Simulation. January,11-14, 1998, San Diego, California. Disponível em http://www.cise.ufl.edu/ fishwick/webconf.html . Acesso em novembro de 2008. 


\section{Anexo I - Web-Based simulation: Pesquisas e aplicações}

\begin{tabular}{|c|c|c|c|}
\hline & Artigo & Autor & Instituição \\
\hline WSC & $\begin{array}{l}\text { Web-Based Simulation: Some Personal } \\
\text { Observations }\end{array}$ & Paul A. Fishwick & University of Florida \\
\hline WSC & $\begin{array}{l}\text { Discrete-Event Simulation on the World } \\
\text { Wide Web Using Java }\end{array}$ & $\begin{array}{l}\text { Arnold H. Buss, Kirk A. } \\
\text { Stork }\end{array}$ & $\begin{array}{ll}\text { Naval } & \text { Postgraduate } \\
\text { School } & \end{array}$ \\
\hline WSC & $\begin{array}{l}\text { A Java-Based Query } \text { Driven Simulation } \\
\text { Environment }\end{array}$ & $\begin{array}{l}\text { Rajesh S. Nair, John A. } \\
\text { Miller, Zhiwei Zhang }\end{array}$ & University of Georgia \\
\hline WSC & $\begin{array}{l}\text { Web-Based Simulation in Simjava Using } \\
\text { Remote Method Invocation }\end{array}$ & $\begin{array}{l}\text { Ernest Page, Robert } \\
\text { Moose, Sean Griffin }\end{array}$ & The Mitre Corporation \\
\hline WSC & An Architectural Design for Digital Objects & Paul A. Fishwick & University of Florida \\
\hline WSC & $\begin{array}{l}\text { Component-Based Simulation Environments: } \\
\text { JSIM as a Case Study using Java Beans }\end{array}$ & $\begin{array}{l}\text { John A. Miller, Yongfu } \\
\text { Ge, Junxin Tao }\end{array}$ & University of Georgia \\
\hline WSC & $\begin{array}{l}\text { A Widely Deployable Web-Based Network } \\
\text { Simulation Framework Using CORBA IDL- } \\
\text { Based APIs }\end{array}$ & $\begin{array}{l}\text { Arjun Cholkar } \\
\text { Philip Koopman }\end{array}$ & $\begin{array}{l}\text { GTE Data Services } \\
\text { Carnegie Mellon }\end{array}$ \\
\hline WSC & $\begin{array}{l}\text { On-Line Data Processing in Simulation } \\
\text { Models: New Approaches and Possibilities } \\
\text { through HLA }\end{array}$ & $\begin{array}{l}\text { Thomas Schulze, Steffen } \\
\text { Strassbu } \\
\text { Ulrich Klein }\end{array}$ & $\begin{array}{l}\text { Otto-von-Guericke- } \\
\text { Universität Magdeburg } \\
\text { Fraunhofer Institute for } \\
\text { Factory Operation and } \\
\text { Automation }\end{array}$ \\
\hline WSC & $\begin{array}{l}\text { Web-Based Performance Visualization of } \\
\text { Distributed Discrete Event Simulations }\end{array}$ & $\begin{array}{l}\text { Adel S. Elmaghraby, } \\
\text { Sherif Elfayoumy, Irfan } \\
\text { Karachiwala, James H. } \\
\text { Graham, Ahmed Emam, } \\
\text { and AlaaEldin Sleem }\end{array}$ & University of Louisville \\
\hline WSC & $\begin{array}{lll}\text { Distributed } & \text { Web-Based } & \text { Simulation } \\
\text { Optimization } & & \end{array}$ & $\begin{array}{l}\text { Yuh-Chyun Luo } \\
\text { Chun-Hung Chen } \\
\text { Enver Yücesan } \\
\text { Insup Lee } \\
\end{array}$ & $\begin{array}{l}\text { Chung-Cheng Institute of } \\
\text { Technology } \\
\text { George Mason University } \\
\text { INSEAD } \\
\text { University } \\
\text { Pennsylvania }\end{array}$ \\
\hline WSC & $\begin{array}{l}\text { The Monarc Toolset for Simulating Large } \\
\text { Network-Distributed Processing Systems }\end{array}$ & $\begin{array}{l}\text { Iosif C. Legrand and } \\
\text { Harvey B. Newman }\end{array}$ & $\begin{array}{l}\text { California Institute of } \\
\text { Technology }\end{array}$ \\
\hline WSC & $\begin{array}{l}\text { A Web-Based Interface for Storing and } \\
\text { Executing Simulation Models }\end{array}$ & $\begin{array}{l}\text { Ashu Guru, Paul Savory, } \\
\text { and Robert Williams }\end{array}$ & University of Nebraska \\
\hline WSC & $\begin{array}{l}\text { A Java-Based Simulation Manager for Web- } \\
\text { Based Simulation }\end{array}$ & $\begin{array}{l}\text { Charles Marr and } \\
\text { Christopher Storey } \\
\text { William E. Biles } \\
\text { Jack P.C. Kleijnen }\end{array}$ & $\begin{array}{l}\text { US Army } \\
\text { University of Louisville } \\
\text { Tilburg University }\end{array}$ \\
\hline WSC & $\begin{array}{l}\text { An Interactive Land Use VRML Application } \\
\text { (ILUVA) with Servlet Assist }\end{array}$ & $\begin{array}{l}\text { Lee A. Belfore, II and } \\
\text { Suresh Chititho }\end{array}$ & Old Dominion University \\
\hline WSC & $\begin{array}{l}\text { A Model-Based Approach for Component } \\
\text { Simulation Development }\end{array}$ & $\begin{array}{l}\text { Perakath Benjamin, } \\
\text { Dursun Delen, and } \\
\text { Richard Mayer } \\
\text { Timothy O'Brien }\end{array}$ & $\begin{array}{l}\text { Knowledge } \\
\text { Systems } \\
\text { NASA }\end{array}$ \\
\hline
\end{tabular}




\begin{tabular}{|c|c|c|c|}
\hline WSC & $\begin{array}{l}\text { Dynamic Component Substitution in Web- } \\
\text { Based Simulation }\end{array}$ & $\begin{array}{l}\text { Dhananjai Madhava Rao } \\
\text { and Philip A. Wilsey }\end{array}$ & University of Cincinnati \\
\hline WSC & $\begin{array}{l}\text { Finding a Substrate for } \text { Federated } \\
\text { Components on the Web }\end{array}$ & $\begin{array}{l}\text { John A. Miller, Andrew } \\
\text { F. Seila, and Junxiu Tao }\end{array}$ & University of Georgia \\
\hline WSC & $\begin{array}{l}\text { SIMFONE': An Object-Oriented Simulation } \\
\text { Framework }\end{array}$ & $\begin{array}{l}\text { Manuel D. Rossetti } \\
\text { Ben Aylor, Ryan } \\
\text { Jacoby, Alyson Prorock, } \\
\text { and Antoine White Ben } \\
\text { Aylor, Ryan Jacoby, } \\
\text { Alyson Prorock, and } \\
\text { Antoine White }\end{array}$ & $\begin{array}{l}\text { University of Arkansas } \\
\text { University of Virginia }\end{array}$ \\
\hline WSC & $\begin{array}{l}\text { VisualSLX - An Open User Shell for High- } \\
\text { Performance Modeling and Simulation }\end{array}$ & Thomas Wiedemann & $\begin{array}{l}\text { Technical University of } \\
\text { Berlin }\end{array}$ \\
\hline WSC & $\begin{array}{l}\text { A Review of Web Based Simulation: } \\
\text { Whither We Wander? }\end{array}$ & $\begin{array}{l}\text { Jasna Kuljis and Ray } \mathrm{J} . \\
\text { Paul }\end{array}$ & Brunel University \\
\hline WSC & $\begin{array}{l}\text { Issues in Java-Based Continuous Time Step } \\
\text { Physical Modelling }\end{array}$ & $\begin{array}{l}\text { Lisa A. Schaefer } \\
\text { Philip M. Wolfe }\end{array}$ & $\begin{array}{l}\text { The MITRE Corporation } \\
\text { Arizona State University }\end{array}$ \\
\hline WSC & $\begin{array}{l}\text { Open Source Simulation Modeling Language } \\
\text { (SML) }\end{array}$ & $\begin{array}{l}\text { Richard A. Kilgore } \\
\text { Dean C. Chatfiel }\end{array}$ & $\begin{array}{l}\text { ThreadTec } \\
\text { Virginia Tech }\end{array}$ \\
\hline WSC & $\begin{array}{l}\text { SISCO: A Supply Chain Simulation Tool } \\
\text { Utilizing Silk }{ }^{\mathrm{TM}} \text { and XML }\end{array}$ & $\begin{array}{l}\text { Terry P. Harrison and } \\
\text { Jack C. Hayya }\end{array}$ & Penn State University \\
\hline WSC & $\begin{array}{l}\text { Web-Based Simulation of Systems Described } \\
\text { by Partial Differential Equations }\end{array}$ & $\begin{array}{l}\text { Manuel Alfonseca } \\
\text { Juan de Lara and Hans } \\
\text { Vangheluwe }\end{array}$ & $\begin{array}{l}\text { Univsersidad Autonoma } \\
\text { Madrid } \\
\text { Juan de Lara and Hans } \\
\text { Vangheluwe }\end{array}$ \\
\hline WSC & $\begin{array}{l}\text { Managing Event Traces for a Web Front-End } \\
\text { to a Parallel Simulation }\end{array}$ & $\begin{array}{l}\text { Boon Ping Gan, Li Liu, } \\
\text { and Zhengrong Ji } \\
\text { Stephen J. Turner and } \\
\text { Wentong Cai }\end{array}$ & $\begin{array}{l}\text { Gintic Institute of } \\
\text { Manufacturing } \\
\text { Technology } \\
\text { Nanyang Technological } \\
\text { University Nanyang } \\
\text { Technological University }\end{array}$ \\
\hline WSC & $\begin{array}{l}\text { The Design of a Web-Based Training System } \\
\text { for Simulation Analysis }\end{array}$ & $\begin{array}{l}\text { Yu-Hui Tao and Shin- } \\
\text { Ming Guo }\end{array}$ & I-Shou University \\
\hline WSC & $\begin{array}{l}\text { D-SOL; A Distributed Java based Discrete } \\
\text { Event Simulation Architecture }\end{array}$ & $\begin{array}{l}\text { Peter H.M. Jacobs, Niels } \\
\text { A. Lang, and Alexander } \\
\text { Verbraeck }\end{array}$ & $\begin{array}{l}\text { Delft University of } \\
\text { Technology }\end{array}$ \\
\hline WSC & $\begin{array}{l}\text { Transaction Cycle of Agents and Web-Based } \\
\text { Gaming Simulation for International } \\
\text { Emissions Trading }\end{array}$ & $\begin{array}{l}\text { Hideyuki Mizuta } \\
\text { Yoshiki Yamagata }\end{array}$ & $\begin{array}{l}\text { IBM Japan } \\
\text { National Institute for } \\
\text { Environmental Studier }\end{array}$ \\
\hline WSC & $\begin{array}{l}\text { Web based Simulation Center: Professional } \\
\text { Support for Simulation Projects }\end{array}$ & $\begin{array}{l}\text { James O. Henriksen } \\
\text { Peter Lorenz } \\
\text { André Hanisch } \\
\text { Stefan Osterburg } \\
\text { Thomas Schriber }\end{array}$ & $\begin{array}{l}\text { Wolverine Corporation } \\
\text { Otto-von-Guericke- } \\
\text { Universität Magdeburg } \\
\text { Fraunhofer Institut für } \\
\text { Fabrikbetrieb und } \\
\text { Automatisierung } \\
\text { University of Michigan }\end{array}$ \\
\hline
\end{tabular}




\begin{tabular}{|c|c|c|c|}
\hline WSC & $\begin{array}{l}\text { Configuration, Simulation and Animation of } \\
\text { Manufacturing Systems via the Internet }\end{array}$ & $\begin{array}{l}\text { Tom-David Graupner, } \\
\text { Hendrik Richter, and } \\
\text { Wilfried Sihn }\end{array}$ & $\begin{array}{l}\text { Fraunhofer Institut fur } \\
\text { Produktionstechnik und } \\
\text { Automatisierung }\end{array}$ \\
\hline WSC & $\begin{array}{l}\text { The ABELS System: Designing an } \\
\text { Adaptable Interface for Linking Simulations }\end{array}$ & $\begin{array}{l}\text { G. Ayorkor Mills-Tettey, } \\
\text { Greg Johnston, Linda F. } \\
\text { Wilson, Joseph M. } \\
\text { Kimpel, and Bin Xie }\end{array}$ & Dartmouth College \\
\hline WSC & $\begin{array}{l}\text { Simulation Web Services with } \text {.Net } \\
\text { Technologies }\end{array}$ & Richard A. Kilgore & $\begin{array}{l}\text { OpenSML and } \\
\text { ThreadTec, Inc }\end{array}$ \\
\hline WSC & Re-Introducing Web-Based Simulation & Steven W. Reichenthal & Boeing \\
\hline WSC & $\begin{array}{l}\text { Implementing the High Level Architecture in } \\
\text { the Virtual Test Bed }\end{array}$ & $\begin{array}{l}\text { José A. Sepúlveda, Luis } \\
\text { Rabelo, and Jaebok Park } \\
\text { Frank Riddick } \\
\text { Cary Peaden }\end{array}$ & $\begin{array}{l}\text { University of Central } \\
\text { Florida } \\
\text { National Institute } \\
\text { Standards } \\
\text { Technology } \\
\text { NASA }\end{array}$ \\
\hline WSC & $\begin{array}{l}\text { Parallel Discrete Event Simulation of Space } \\
\text { Shuttle Operations }\end{array}$ & $\begin{array}{l}\text { Luis Rabelo, José } \\
\text { Sepúlveda, Mario Marin, } \\
\text { Amith Paruchuri, Amit } \\
\text { Wasadikar, and Karthik } \\
\text { Nayaranan }\end{array}$ & $\begin{array}{l}\text { University of Central } \\
\text { Florida }\end{array}$ \\
\hline WSC & $\begin{array}{l}\text { Efficient Process Interaction Simulation in } \\
\text { Java: Implementing Co-Routines Within a } \\
\text { Single Java Thread }\end{array}$ & $\begin{array}{l}\text { Richard M. Weatherly } \\
\text { and Ernest H. Page }\end{array}$ & The MITRE Corporation \\
\hline WSC & $\begin{array}{l}\text { SRML Case Study: Simple Self-Describing } \\
\text { Process Modeling and Simulation }\end{array}$ & Steven W. Reichenthal & Boeing \\
\hline WSC & $\begin{array}{l}\text { XML Mediation Services Utilizing Model } \\
\text { Based Data Management }\end{array}$ & Andreas Tolk & Old Dominion University \\
\hline WSC & $\begin{array}{l}\text { Examining the Feasibility of Constructing } \\
\text { Simulation Models Using the Web-Based } \\
\text { 'Grab-and-Glue' Framework }\end{array}$ & $\begin{array}{l}\text { Tillal Eldabi, Man Wai } \\
\text { Lee, and Ray J. Paul }\end{array}$ & Brunel University \\
\hline WSC & $\begin{array}{l}\text { HDPS, an XML/XSLT Based Hierarchal } \\
\text { Modeling System }\end{array}$ & $\begin{array}{l}\text { Richard Evan Curry } \\
\text { Kikiakos Vlahos }\end{array}$ & $\begin{array}{l}\text { London Business School } \\
\text { Athens Laboratory of } \\
\text { Business Administration }\end{array}$ \\
\hline WSC & $\begin{array}{l}\text { XML-Based Supply Chain } \text { Simulation } \\
\text { Modeling }\end{array}$ & $\begin{array}{l}\text { Dean C. Chatfield } \\
\text { Terry P. Harrison and } \\
\text { Jack C. Hayya }\end{array}$ & $\begin{array}{l}\text { Virginia Tech } \\
\text { Penn State }\end{array}$ \\
\hline WSC & $\begin{array}{l}\text { Simulation of Grid Computing Infrastructure: } \\
\text { Challenges and Solutions }\end{array}$ & Sugato Bagchi & $\begin{array}{lcc}\text { IBM } & \text { T. J. } & \text { Watson } \\
\text { Research Center } & \end{array}$ \\
\hline WSC & $\begin{array}{l}\text { Time Management in Distributed Factory } \\
\text { Simulation, a Case Study Using HLA }\end{array}$ & $\begin{array}{l}\text { Ke Wang, Sheng Xu, } \\
\text { and Leon F. McGinnis }\end{array}$ & $\begin{array}{l}\text { Georgia Institute of } \\
\text { Technology }\end{array}$ \\
\hline WSC & Initialization of Online Simulation Models & $\begin{array}{lr}\text { Andre } & \text { Hanisc, Juri } \\
\text { Tolujew } & \text { Thomas } \\
\text { Schulze } & \\
\end{array}$ & $\begin{array}{l}\text { Fraunhofer Institute IFF } \\
\text { University of Magdeburg }\end{array}$ \\
\hline WSC & $\begin{array}{l}\text { Addressing Complexity Using Distributed } \\
\text { Simulation: A Case Study in Spaceport } \\
\text { Modeling }\end{array}$ & $\begin{array}{l}\text { Jaebok Park, Reinaldo } \\
\text { Moraga, Luis Rabelo, } \\
\text { Jeffrey W. Dawson, } \\
\text { Mario Marin e Jose } \\
\text { Sepulveda } \\
\text { Mathias Roehl e Adelind } \\
\text { M. Uhrmacher }\end{array}$ & $\begin{array}{l}\text { University of Central } \\
\text { Florida }\end{array}$ \\
\hline
\end{tabular}




\begin{tabular}{|c|c|c|c|}
\hline $\begin{array}{l}\text { ANS } \\
\text { S }\end{array}$ & $\begin{array}{l}\text { Building a Web-Based Federated Simulation } \\
\text { System with Jini and XML } \\
\text { Using simulation to facilitate effective } \\
\text { workflow adaptation }\end{array}$ & $\begin{array}{l}\text { Xuexim Huag } \\
\text { John A. Miller } \\
\text { J. A. Miller e Jorge } \\
\text { Cardoso }\end{array}$ & University of Georgia \\
\hline $\begin{array}{l}\text { ANS } \\
\text { S }\end{array}$ & $\begin{array}{l}\text { Evolving The Web-Based distributed } \\
\text { SI/PDO Architecture for High-Performance } \\
\text { Visualization }\end{array}$ & $\begin{array}{l}\text { V.P. Holmes, J.M. } \\
\text { Linabarger, D.J. Miller e } \\
\text { R.L. Vanderwart } \\
\text { C.P. Crowley }\end{array}$ & $\begin{array}{l}\text { Sandia National } \\
\text { Laboratories } \\
\text { University of New } \\
\text { Mexico }\end{array}$ \\
\hline $\begin{array}{l}\text { ANS } \\
\text { S }\end{array}$ & $\begin{array}{l}\text { Multiuser Extensions to the interactive Land } \\
\text { Use VRML application (ILUVA) }\end{array}$ & $\begin{array}{l}\text { Lee A. Belfore e S. } \\
\text { Chitithoti }\end{array}$ & Old Dominium University \\
\hline $\begin{array}{l}\text { ANS } \\
\text { S }\end{array}$ & $\begin{array}{l}\text { An architecture and implementation to } \\
\text { support large-scale data access in scientific } \\
\text { simulation environments }\end{array}$ & $\begin{array}{l}\text { V. P. Holmes, S.Kleban, } \\
\text { D.J.Miller, C. Pavlakos, } \\
\text { C. Poore e R.L. } \\
\text { Vanderwart } \\
\text { C.P. Crowley }\end{array}$ & $\begin{array}{l}\text { Sandia National } \\
\text { Laboratories } \\
\begin{array}{l}\text { University of New } \\
\text { Mexico }\end{array}\end{array}$ \\
\hline $\begin{array}{l}\text { ANS } \\
\text { S }\end{array}$ & $\begin{array}{l}\text { GroupGraph: A collaborative hierarquical } \\
\text { graph editor based on the internet } \\
\text { Translating activity cycle diagrams to java } \\
\text { programs }\end{array}$ & $\begin{array}{l}\text { H. A.S. Lima Filho } \\
\text { Celso M. Hirata } \\
\text { Wladimir L. Araujo } \\
\text { Filho e Celso M. Hirata }\end{array}$ & $\begin{array}{lr}\text { ITA } \quad- & \text { Instituto } \\
\text { Tecnológico } & \text { de } \\
\text { Aeronáutica } & \end{array}$ \\
\hline $\begin{array}{l}\text { ANS } \\
\text { S }\end{array}$ & $\begin{array}{l}\text { Performance simulation of the Jambala } \\
\text { Platform }\end{array}$ & Maria Toeroe & Ericson Research \\
\hline $\begin{array}{l}\text { ANS } \\
\text { S }\end{array}$ & $\begin{array}{l}\text { Integrating Web service and grid Enabling } \\
\text { technologies to provide desktop access to } \\
\text { high-performance components for large-scale } \\
\text { data services }\end{array}$ & $\begin{array}{l}\text { Victor P. Holmes, } \\
\text { Wilbur R. Johnson e } \\
\text { David J. Miller }\end{array}$ & $\begin{array}{ll}\text { Sandia } & \text { National } \\
\text { Laboratories } & \end{array}$ \\
\hline $\begin{array}{l}\text { ANS } \\
\text { S }\end{array}$ & $\begin{array}{l}\text { Computational Science and engineering on- } \\
\text { line: an integrated web-based simulation } \\
\text { environment for computational science and } \\
\text { engineering }\end{array}$ & T. n. Truong & University of Utah \\
\hline $\begin{array}{l}\text { ANS } \\
\text { S }\end{array}$ & $\begin{array}{l}\text { A self-manageable infrastructure for } \\
\text { supporting web-based simulations } \\
\text { Autonomic Web-Based simulation }\end{array}$ & $\begin{array}{l}\text { Y. Huang, X. Xiang e G. } \\
\text { Madey } \\
\text { Y. Huang e G. Madey }\end{array}$ & University of Notre Dame \\
\hline $\begin{array}{l}\text { ORA } \\
\text { HS }\end{array}$ & A multi-user simulation of a hospital queue & $\begin{array}{l}\text { Adriana B. Moraes, } \\
\text { Mário J. F. de Oliveira, } \\
\text { Sheila M. Esposito e } \\
\text { Simone M. Bordalo }\end{array}$ & $\begin{array}{l}\text { Federal University of Rio } \\
\text { de Janeiro }\end{array}$ \\
\hline
\end{tabular}

Fonte: Winter simulation Conference - WSC (1996-2005), Annual Simulation Symposium ANSS (2001-2005) e Operational Research Applied to Health Services 